\title{
Termination Conditions for Positivity Proving Procedures
}

\author{
Veronika Pillwein ${ }^{*}$ \\ RISC-Linz \\ Johannes Kepler University \\ 4040 Linz (Austria) \\ vpillwei@risc.jku.at
}

\begin{abstract}
Proving positivity of a sequence given by a linear recurrence with polynomial coefficients ( $\mathrm{P}$-finite recurrence) is a non-trivial task for both humans and computers. Algorithms dealing with this task are rare or non-existent. One method that was introduced in the last decade by Gerhold and Kauers succeeds on many examples, but termination of this procedure has been proven so far only up to order three for special cases. Here we present an analysis that extends the previously known termination results on recurrences of order three, and also provides termination conditions for recurrences of higher order.
\end{abstract}

\section{Categories and Subject Descriptors}

I.1.2 [Computing Methodologies]: Symbolic and Algebraic Manipulation-Algorithms; G.2.1 [Discrete Mathematics]: Combinatorics-Recurrences and difference equations

\section{General Terms}

Algorithms

\section{Keywords}

P-finite Sequences, Positivity, Cylindrical decomposition

\section{INTRODUCTION}

Special functions are interesting mathematical objects, in particular from the point of view of symbolic computation. They are often defined by linear recurrences, differential equations, or mixed difference-differential equations which makes them a suitable input for several computer algebra methods. Nowadays, a multitude of algorithms is available that can automatically prove or derive transformations, identities or closed forms for special functions.

*Supported by the Austrian Science Fund (FWF) grant P22748-N18.

Permission to make digital or hard copies of all or part of this work for personal or classroom use is granted without fee provided that copies are not made or distributed for profit or commercial advantage and that copies bear this notice and the full citation on the first page. To copy otherwise, to republish, to post on servers or to redistribute to lists, requires prior specific permission and/or a fee.

ISSAC 2013, 26-29 June 2013, Boston, USA.

Copyright 2013 ACM .....\$10.00.
The situation is quite different for special functions inequalities, which arise in many applications in mathematics and physics. These are still a serious challenge for both humans and computers. Even for special cases such as sequences satisfying linear recurrence relations with constant coefficients, deciding whether the given sequence is positive leads to hard number theoretic questions to which no solutions are known today $[12,13]$.

Despite these difficulties, some progress has been made in the last decade. A few years ago, Mezzarobba and Salvy have given an algorithm for effectively computing tight upper bounds for sequences defined by linear recurrences with polynomial coefficients [22]. Recently, Cha [6] has extended a method for finding closed form solutions of difference equations introduced by Cha, van Hoeij, and Levy [7] to a method that determines closed form solutions that are linear combinations of sums of squares. In the lucky case, it suffices to prove positivity of the rational function coefficients using, e.g., Cylindrical Algebraic Decomposition [9, 10, 5, 2] (CAD), to conclude positivity of the given sequence.

In 2005, Gerhold and Kauers [14] proposed a method that is applicable to proving inequalities concerning sequences that satisfy recurrence equations of a very general type. Their method consists of constructing a sequence of polynomial sufficient conditions that would imply the non-polynomial inequality under consideration. The truth of these conditions can be detected for instance using CAD. If the inequality does not hold, then the method terminates after a finite number of steps and returns a counterexample. If the inequality holds, then either the program terminates and returns True or it may fail to detect this and run forever.

Despite its simplicity, the method has been quite successful in applications. Not only did it provide the first computer proofs of some special function inequalities from the literature $[14,15,18,19]$, but it even helped to resolve some open conjectures $[1,20,19,23]$. At the same time, the lack of termination conditions was unsatisfactory from a computational point of view. First results on a priori conditions, for some restricted classes, guaranteeing that the method (or some variation of it) will succeed were determined only recently [21] for sequences defined by linear recurrence equations with polynomial coefficients (P-finite sequences) up to order three.

The aim of this paper is to extend these known termination results for the original proving procedure introduced by Gerhold and Kauers (Algorithm 1 below) for recurrences of order three and to obtain new termination conditions for recurrences of higher order. As auxiliary tool, we propose a 
new variation of the method (Algorithm 3 below) for proving positivity of $\mathrm{P}$-finite sequences. If Algorithm 3 terminates on a given input, then Algorithm 1 also terminates on this input. The new method, however, allows for a different analysis than the initial one which leads to an extension of the known termination conditions (Theorem 2 and Corollary 1 ). Some parts of the analysis are not affected by the order and thus also termination conditions for higher orders are obtained (Corollary 2 and Theorem 3). An interesting aspect of the analysis is that algorithms for real quantifier elimination are not only used in the execution of Algorithm 1 and 3, but also enter in the proof of the termination theorems.

\section{PRELIMINARIES}

A sequence $f: \mathbb{N} \rightarrow K$, where $K$ is a computable subfield of $\mathbb{C}$, is called $P$-finite (or holonomic) if there exist polynomials $p_{0}, \ldots, p_{d} \in K[x]$, not all zero, such that

$$
p_{0}(n) f(n)+p_{1}(n) f(n+1)+\cdots+p_{d}(n) f(n+d)=0
$$

for all $n \in \mathbb{N}$. Such an equation is called a (P-finite) recurrence, and $d$ is called its order if $p_{0}$ and $p_{d}$ are not vanishing entirely. In the special case when all coefficients in the recurrence are from the ground field $K$ we call it a $C$-finite recurrence. If $p_{d}(n) \neq 0$ for all $n \in \mathbb{N}$, then the infinite sequence $f$ is uniquely determined by the recurrence and $d$ initial values $f(0), f(1), \ldots, f(d-1)$. In this case, we may also rewrite the recurrence with rational function coefficients as

$$
f(n+d)=r_{d-1}(n) f(n+d-1)+\cdots+r_{0}(n) f(n),
$$

with $r_{k}(n)=-\frac{p_{k}(n)}{p_{d}(n)}$. The assumption $p_{d}(n) \neq 0$ for all $n \in \mathbb{N}$ can be adopted without loss of generality, because we can substitute $g(n)=f(n+N)$ for some $N$ larger than the biggest integer root of $p_{d}$ and then consider $g$ instead of $f$ and check non-negativity of the finitely many terms $f(0), \ldots, f(N-1)$ by inspection.

A P-finite recurrence is called balanced if $\operatorname{deg} p_{0}=\operatorname{deg} p_{d}$ and $\operatorname{deg} p_{i} \leq \operatorname{deg} p_{0}(i=1, \ldots, d)$. The characteristic polynomial of a balanced recurrence is defined as

$\chi(x)=\mathrm{lc}_{y}\left(p_{0}(y)+p_{1}(y) x+p_{2}(y) x^{2}+\cdots+p_{d}(y) x^{d}\right) \in \mathbb{Q}[x]$.

Its roots $\alpha_{1}, \ldots, \alpha_{d} \in \mathbb{C}$ are called the eigenvalues of the recurrence. (The $\alpha_{i}$ are not necessarily distinct.) Note that for a balanced recurrence, the characteristic polynomial has always degree $d$ and it has never 0 as a root.

An eigenvalue $\alpha_{i}$ is called dominant if $\left|\alpha_{j}\right| \leq\left|\alpha_{i}\right|$ for all $j=1, \ldots, d$. If none of the dominant eigenvalues $\alpha_{i}$ is real and positive, then it is clear that $f$ will be ultimately oscillating [3] (if the recurrence is of minimal order), and so $f(n) \geq 0$ cannot possibly be true for all $n$. This case can be sorted out trivially beforehand, and we may therefore assume that there is a real and positive dominant eigenvalue. In that case, if $\alpha_{i} \neq 1$, we consider the P-finite sequence $g(n)=f(n) / \alpha_{i}^{n}$ instead, whose dominant eigenvalue is 1 . Since $f(n) \geq 0 \Leftrightarrow g(n) \geq 0$ it suffices to consider the case when $\alpha_{i}=1$ for some $i$ and $\left|\alpha_{j}\right| \leq\left|\alpha_{i}\right|$ for all $j \neq i$.

\section{INDUCTION BASED PROVING PROCEDURES}

Let $f(n)$ be a P-finite sequence order $d$ given in terms of its defining recurrence relation and initial values. Based on this information it is to be decided whether the sequence is non-negative for all $n \geq 0$.

\subsection{Summary of previous algorithms}

The initial version of the algorithm as introduced by Gerhold and Kauers [14] proceeds by induction:

$$
\begin{aligned}
& f(n) \geq 0 \wedge \cdots \wedge f(n+d-1) \geq 0 \Longrightarrow \\
& r_{d-1}(n) f(n+d-1)+\cdots+r_{1}(n) f(n+1)+r_{0}(n) f(n) \geq 0
\end{aligned}
$$

A sufficient condition for this to hold for all $n \in \mathbb{N}$ is that the induction step formula

$$
\begin{aligned}
& \forall y_{0}, y_{1}, \ldots, y_{d-1} \in \mathbb{R} \forall x \in \mathbb{R}: \\
& \quad\left(x \geq 0 \wedge y_{0} \geq 0 \wedge \cdots \wedge y_{d-1} \geq 0\right) \\
& \quad \Longrightarrow r_{d-1}(x) y_{d-1}+\cdots+r_{0}(x) y_{0} \geq 0 .
\end{aligned}
$$

is true, and this can be decided by a quantifier elimination algorithm. If it is true, the induction step is established and $f$ is non-negative everywhere if and only if it is nonnegative for $n=0, \ldots, d-1$, which can be checked. Passing from discrete values of $n$ to a real variable $x$ (and possibly ignoring other relevant information passing from $f(n+i)$ to real variables $y_{i}$ ), the induction step formula may be false even though the given sequence is non-negative. If the formula is false, then in the next step the induction hypothesis is extended and the formulas

$$
f(n) \geq 0 \wedge \cdots \wedge f(n+D-1) \geq 0 \Longrightarrow f(n+D) \geq 0
$$

for $D>d$ are constructed. Using the recurrence, each term $f(n+i)$ can be rewritten as a linear combination of $f(n)$, $\ldots, f(n+d-1)$ with rational function coefficients, and using this rewriting, refined induction step formulas are built:

$$
\begin{aligned}
\Phi(D):= & \forall y_{0}, y_{1}, \ldots, y_{d-1} \in \mathbb{R} \forall x \in \mathbb{R}: \\
& \left(x \geq 0 \wedge y_{0} \geq 0 \wedge \cdots \wedge y_{d-1} \geq 0\right. \\
& \wedge R_{0}(d, x) y_{0}+\cdots+R_{d-1}(d, x) y_{d-1} \geq \\
& \wedge R_{0}(d+1, x) y_{0}+\cdots+R_{d-1}(d+1, x) y_{d-1} \geq 0 \\
& \vdots \\
& \left.\wedge R_{0}(D-1, x) y_{0}+\cdots+R_{d-1}(D-1, x) y_{d-1} \geq 0\right) \\
& \Rightarrow R_{0}(D, x) y_{0}+\cdots+R_{d-1}(D, x) y_{d-1} \geq 0,
\end{aligned}
$$

where the $R_{i}(j, \cdot)$ are some rational functions. The full method then reads as follows.

\section{Algorithm 1.}

Input: A P-finite recurrence of order $d$ and a vector of initial values defining a sequence $f: \mathbb{N} \rightarrow \mathbb{Q}$.

Output: True if $f(n) \geq 0$ for all $n \in \mathbb{N}$, False if $f(n)<0$ for some $n \in \mathbb{N}$, possibly no output at all.

$$
\begin{aligned}
& 1 \text { for } n=0 \text { to } d-1 \underline{\text { do }} \\
& 2 \text { if } f(n)<0 \text { then return False } \\
& 3 \text { for } n=d, d+1, d+2, d+3, \ldots \text { do } \\
& 4 \text { if } \Phi(n) \text { then return True } \\
& 5 \quad \text { if } f(n)<0 \text { then return False }
\end{aligned}
$$

In [21] a variation of Algorithm 1 was introduced that covers some of its non-terminating cases. The basic idea is to determine a constant factor $M>0$ such that the sequence $M^{n} f(n)$ is increasing and such that this fact can be proven by an application of Algorithm 1 without extending the induction hypothesis. We refer to this algorithm as 
Algorithm 2. Termination for both variants has been investigated up to recurrences of order three [21]. These results are summarized in the figure below in terms of the coefficients of the characteristic polynomial $u_{1}, u_{0}$, where

$$
\chi(x)=(x-1)\left(x^{2}+u_{1} x+u_{0}\right) .
$$

The outer triangle corresponds to the area where the roots are in the interior of the complex unit disk, the gray shaded area is the termination region of Algorithm 2, and the dashed areas are terminating regions for Algorithm 1. For the latter, the parts $I_{D}$ correspond to induction hypotheses of length $D$.

In particular, the region where Algorithm 1 terminates in the first step is $I_{3}=\left\{\left(u_{1}, u_{0}\right) \mid 0<u_{0}<u_{1}<1\right\}$. This corresponds to the trivial case when all coefficients in the recurrence eventually are positive.

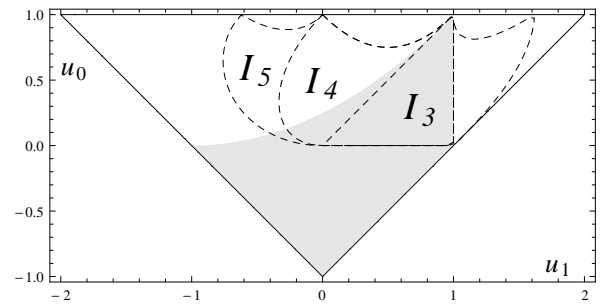

For an induction hypothesis of length three, i.e., the case when the induction succeeds in the first step, the algorithm provably terminates only in the case when all the coefficients of the characteristic polynomial are non-negative. Induction hypotheses of length six and more as well as higher order cases have not been discussed in the previous paper because of computational limitations.

\subsection{Algorithm 3}

Our aim is to extend the known termination results for Algorithm 1 and our tool in the analysis is a variant of it. This new method, Algorithm 3, has the property that if it terminates then also the original algorithm terminates, but it allows to obtain better statements about the terminating region. The efficiency of Algorithm 3 is not investigated.

Let $f(n)$ be a given P-finite sequence of order $d$. Instead of proceeding by induction on the sequence itself, we consider the shifted subsequences $f(n+m)$, for some fixed natural number $m \geq d$, and aim at proving inductively that nonnegativity of $d$ successive sequence elements implies nonnegativity of one such shifted sequence. That is, we prove that

$f(n) \geq 0 \wedge f(n+1) \geq 0 \wedge \cdots \wedge f(n+d-1) \geq 0 \Rightarrow f(n+m) \geq 0$,

for $n \geq n_{0}$, for some lower bound $n_{0} \geq 0$. Using the recurrence relation, $f(n+m)$ can be expressed as linear combination of $f(n), \ldots, f(n+d-1)$ with rational function coefficients. Checking initial values $f(0) \geq 0, \ldots, f(m-1) \geq 0$ completes the proof. The (modified) refined induction step formula for this variation reads as

$$
\begin{aligned}
& \widetilde{\Psi}(m):= \xi \forall y_{0}, y_{1}, \ldots, y_{d-1} \in \mathbb{R} \forall x \in \mathbb{R}: \\
&\left(x \geq \xi \geq 0 \wedge y_{0} \geq 0 \wedge \cdots \wedge y_{d-1} \geq 0\right) \\
& \quad \Longrightarrow R_{0}(m, x) y_{0}+\cdots+R_{d-1}(m, x) y_{d-1} \geq 0
\end{aligned}
$$

where the $R_{i}(m, \cdot)$ are some rational functions. In order to determine the lower bound $\xi$ we perform quantifier elimination on the formula where we drop the first quantifier in $\widetilde{\Psi}(m)$. The full method then reads as follows.

\section{Algorithm 3}

Input: A P-finite recurrence of order $d$ and a vector of initial values defining a sequence $f: \mathbb{N} \rightarrow \mathbb{Q}$.

Output: True if $f(n) \geq 0$ for all $n \in \mathbb{N}$, False if $f(n)<0$ for some $n \in \mathbb{N}$, possibly no output at all.

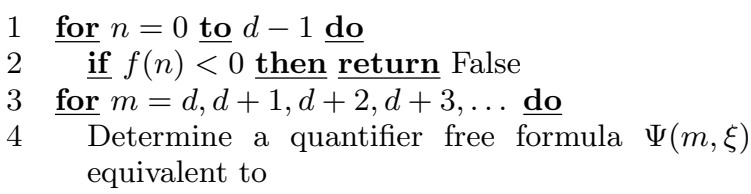

$$
\begin{aligned}
& \xi>0 \wedge \forall x, y_{0}, y_{1}, \ldots, y_{d-1} \in \mathbb{R}: \\
& \left(x \geq \xi \wedge y_{0} \geq 0 \wedge \cdots \wedge y_{d-1} \geq 0\right) \\
& \quad \Rightarrow R_{0}(m, x) y_{0}+\cdots+R_{d-1}(m, x) y_{d-1} \geq 0,
\end{aligned}
$$

$5 \quad$ if $\exists \xi_{0}: \Psi\left(m, \xi_{0}\right)$ then

6 for $n=m, \ldots, m+\left\lceil\xi_{0}\right\rceil-1$ do

$7 \quad$ if $f(n)<0$ then return False

$8 \quad$ return True

9 if $f(m)<0$ then return False

Essentially correctness of this algorithm follows from the correctness of Algorithm 1.

\section{TheOREM 1. Algorithm 3 is correct.}

Proof. Correctness is obvious whenever the algorithm returns False, because this happens only when an explicit point $n$ with $f(n)<0$ has been found. Suppose now that the algorithm returns True at the $m$ th iteration of the for loop. Then $f(0), \ldots, f(m-1)$ are all non-negative, and the condition in line 5 implies inductively for $n_{0}=\left\lceil\xi_{0}\right\rceil$ that

$$
\forall n \geq n_{0}: f(n+m) \geq 0 .
$$

It remains to verify non-negativity for the initial values that have not been checked yet, i.e., that $f(n) \geq 0$ for $m \leq$ $n \leq m+n_{0}-1$. This is carried out in line 7 . Then, for any $d$ consecutive, non-negative terms of the sequence $f(n), \ldots, f(n+d-1)$ with $n \geq n_{0}$, it follows by definition of $\Psi$ and choice of $\xi_{0}$ that $f(n+m) \geq 0$, and thus inductively that $f(n) \geq 0$ for all $n$.

In Algorithm 3 the length of the induction hypothesis remains constantly equal to the order of the recurrence in the refined induction step formula, whereas in Algorithm 1 additional requirements to the hypothesis are added. Since $(A \Rightarrow B) \Rightarrow((A \wedge C) \Rightarrow B)$, termination of Algorithm 3 implies termination of Algorithm 1.

In Algorithm 1 the quantifier free part in the induction step formula is of the form

$$
\mathcal{H}_{d}\left(x, y_{0}, \ldots, y_{d-1}\right) \quad \Longrightarrow \mathcal{C}\left(x, y_{0}, \ldots, y_{d-1}\right) .
$$

Both sides, the hypothesis and the conclusion, define semialgebraic sets and from the geometric point of view it is checked whether the set defined by $\mathcal{H}$ is contained in the set defined by $\mathcal{C}$. If this is not the case, then a further restriction is added to the hypothesis defining a new set that can, if anything, only decrease in size. At the same time the conclusion is altered in order to determine a set large enough to fit the hypothesis. The process is repeated until (in the lucky case) both sides are made fit. On the other hand, Algorithm 3 searches for an appropriate superset to fit the hypothesis. 
Example 1. Let $f: \mathbb{N} \rightarrow K$ be defined by

$$
\begin{aligned}
(20 n+1) f(n+3)= & 3(5 n+1) f(n+2) \\
& -(13 n+1) f(n+1)+(18 n+7) f(n),
\end{aligned}
$$

and $f(0)=f(1)=1, f(2)=3$. The characteristic polynomial of this recurrence has the coefficients $u_{0}=\frac{9}{10}, u_{1}=\frac{1}{4}$ and eigenvalues $1,-\frac{1}{8} \pm \mathbf{i} \frac{\sqrt{1415}}{40}$. These values are chosen as to be outside the previously proven terminating regions.

An application [17] of Algorithm 1 succeeds proving positivity by extending the induction hypothesis up to length nine and also Algorithm 3 succeeds proving positivity for the shifted sequence $f(n+9)$ for $n \geq 0$. In general, there might be a large gap between the length of the induction hypothesis needed for Algorithm 1 and the shift length, hence we do not advertise Algorithm 3 as an alternative for real world problems.

Identifying the terminating region of Algorithm 3 corresponds to identifying the region for which the recurrence coefficients $R_{i}(m, \cdot)$ are in the interior of the terminating region $I_{m}$ of Algorithm 1 for some $m \geq d$. This is made more precise in the next section.

\section{ORDER THREE}

Let $f: \mathbb{N} \rightarrow K$ be a sequence defined by a balanced, Pfinite recurrence of order $d$, where we assume that the leading coefficient has no positive root and we consider the recurrence

$$
f(n+d)=r_{d-1}(n) f(n+d-1)+\cdots+r_{0}(n) f(n),
$$

with rational coefficients $r_{k}(n)=-\frac{p_{k}(n)}{p_{d}(n)}$. The main results in this section are on recurrences of order three, i.e., $d=3$. We assume that 1 is the dominant eigenvalue, and let $\alpha_{k} \in$ $K$ with $\left|\alpha_{k}\right|<1, k=1, \ldots, d-1$, be such that

$$
\chi(x)=(x-1)\left(x-\alpha_{1}\right) \cdots\left(x-\alpha_{d-1}\right)
$$

is the characteristic polynomial of the recurrence. Depending on the context we also use the following alternative representations of the characteristic polynomial

$$
\begin{aligned}
\chi(x) & =x^{d}-c_{d-1} x^{d-1} \cdots-c_{1} x-c_{0} \\
& =(x-1)\left(x^{d-1}+u_{d-2} x^{d-2}+\cdots+u_{0}\right) .
\end{aligned}
$$

The latter allows for direct comparison of the order three results as obtained previously [21]. These three representations are related and we may freely switch between them. For $d=3$, e.g., we have

$$
\begin{aligned}
& u_{0}=c_{0}=\alpha_{1} \alpha_{2}, \\
& u_{1}=c_{0}+c_{1}=-\alpha_{1}-\alpha_{2},
\end{aligned}
$$

and in general, because of $\chi(1)=0$, that

$$
1=c_{0}+c_{1}+\cdots+c_{d-1} .
$$

Repeated application of the recurrence (1) allows to compute $f(n+m)$ (for some $m \geq d$ ) from $d-1$ consecutive sequence elements $f(n), f(n+1), \ldots, f(n+d-1)$,

$$
\begin{aligned}
f(n+m)= & R_{d-1}(m, n) f(n+d-1)+\cdots \\
& \cdots+R_{1}(m, n) f(n+1)+R_{0}(m, n) f(n),
\end{aligned}
$$

where the coefficients $R_{k}(m, \cdot)$ are rational functions. The repeated application of the recurrence acts also on the coefficients $r_{k}(n)$ and it is easy to verify that for $k=0, \ldots, d-1$ they satisfy the recurrence

$$
\begin{aligned}
R_{k}(m+d, n)= & r_{d-1}(n+m) R_{k}(m+d-1, n)+\cdots \\
& \cdots+r_{0}(n+m) R_{k}(m, n), \quad m \geq 0,
\end{aligned}
$$

with initial values $R_{k}(j, n)=\delta_{k, j}$ for all $j=0, \ldots, d-1$ and $k$, where $\delta_{k, j}$ denotes the Kronecker delta. Note that $R_{k}(d, n)=r_{k}(n)$. In particular, for the C-finite recurrence

$$
y(n+d)=c_{d-1} y(n+d-1)+\cdots+c_{1} y(n+1)+c_{0} y(n),
$$

with $c_{k}$ being the coefficients of the characteristic polynomial $\chi(x)$, we have that

$$
\begin{aligned}
y(n+m)= & \gamma_{d-1}(m) y(n+d-1)+\cdots \\
& \cdots+\gamma_{0}(m) y(n), \quad m \geq 0,
\end{aligned}
$$

where the $\gamma_{k}(m)$ satisfy the recurrences

$$
\gamma_{k}(m+d)=c_{d-1} \gamma_{k}(m+d-1)+\cdots+c_{0} \gamma_{k}(m),
$$

with initial values $\gamma_{k}(j)=\delta_{k, j}$ for $j, k=0, \ldots, d-1$. Also these recurrences have characteristic polynomial $\chi(x)$ with largest root 1 and roots $\alpha_{k}$ in the interior of the complex unit disk. Because (3) is a C-finite recurrence, it can be solved explicitly. The general solution is a linear combination of the sequences $(1)_{m \geq 0}$ and sequences of the form

$$
\left(\alpha^{m}\right)_{m \geq 0},\left(m \alpha^{m}\right)_{m \geq 0}, \ldots,\left(m^{e-1} \alpha^{m}\right)_{m \geq 0},
$$

where $\alpha \in\left\{\alpha_{1}, \ldots, \alpha_{d}\right\}$ and $e$ denotes its multiplicity. Since all the roots are strictly less than one in absolute value, the limit for $m$ tending to infinity is given by the coefficient of the eigenvalue 1 .

For the coefficients $r_{k}(n)$ of the defining recurrence of $f(n)$, we have, using the characteristic polynomial, that

$$
\lim _{n \rightarrow \infty} r_{k}(n)=c_{k}, \quad k=0, \ldots, d-1 .
$$

The recurrences (2) and (3) satisfied by the iterated coefficient sequences $R_{k}(m, n)$ and $c_{k}(m)$ are structurally of the same form. This, in combination with the limit relation above, yields that for every fixed $m \geq 0$ we have

$$
\lim _{n \rightarrow \infty} R_{k}(m, n)=\gamma_{k}(m)
$$

With these preliminary considerations at hand, we are in the position to prove that Algorithm 3 terminates, if all the eigenvalues (except 1) have negative real part. Note that up to here we did not put any restrictions on the order $d$. For the first termination result given below, we consider recurrences of order $d=3$. This statement is formulated in terms of the coefficients $u_{0}, u_{1}$ in order to better compare with the previous results.

ThEOREM 2. Algorithm 3 terminates, if $0<u_{1}<2$ and $\max \left(0, u_{1}-1\right)<u_{0}<1$.

Proof. The algorithm terminates if for some $m \geq d$ the coefficients $R_{k}(m, n)$ in the iterated recurrence

$$
\begin{aligned}
f(n+m)= & R_{2}(m, n) f(n+2)+R_{1}(m, n) f(n+1) \\
& +R_{0}(m, n) f(n),
\end{aligned}
$$

are positive which corresponds to the terminating region $I_{3}$ of Algorithm 3. Next, we define the C-finite sequences $\gamma_{k}(m)$, by

$$
\gamma_{k}(m+3)=c_{2} \gamma_{k}(m+2)+c_{1} \gamma_{k}(m+1)+c_{0} \gamma_{k}(m),
$$


with initial values $\gamma_{k}(j)=\delta_{k, j}, k, j=0,1,2$, and denote their respective limits by $\zeta_{k}=\lim _{m \rightarrow \infty} \gamma_{k}(m)$. Then, for any $\epsilon>0$ there is an $m_{0}$ such that for all $m \geq m_{0}$,

$$
\left|\gamma_{k}(m)-\zeta_{k}\right|<\frac{\epsilon}{2}
$$

On the other hand, for every fixed $m_{0}$, the (iterated coefficient) sequences $R_{k}\left(m_{0}, n\right)$ tend to $\gamma_{k}\left(m_{0}\right)$ as $n \rightarrow \infty$, i.e., there exists an $n_{0}$ such that for all $n \geq n_{0}$,

$$
\left|R_{k}\left(m_{0}, n\right)-\gamma_{k}\left(m_{0}\right)\right|<\frac{\epsilon}{2} .
$$

Combining these two estimates using the triangle inequality yields that for any $\epsilon>0$, there exist $m_{0}$ and $n_{0}$ such that for all $n \geq n_{0}$,

$$
\left|R_{k}\left(m_{0}, n\right)-\zeta_{k}\right|<\epsilon .
$$

The proof is completed once we can show that the $\zeta_{k}$ are positive under the assumptions of the theorem.

The C-finite recurrence (4) has the eigenvalues $1, \alpha_{1}, \alpha_{2}$, where either there are two simple roots $\alpha_{1} \neq \alpha_{2}$ (real or complex conjugate), or there is a double root $\alpha=\alpha_{1}=\alpha_{2}$. In the first case the general ansatz for the closed form of the sequence is

$$
\gamma_{k}(m)=\nu_{k}(0)+\nu_{k}(1) \alpha_{1}^{m}+\nu_{k}(2) \alpha_{2}^{m},
$$

in the second case the ansatz is

$$
\gamma_{k}(n)=\nu_{k}(0)+\left(\nu_{k}(1)+m \nu_{k}(2)\right) \alpha^{m} .
$$

The constants $\nu_{k}(0), \nu_{k}(1), \nu_{k}(2)$ are obtained using the initial values $\gamma_{k}(j)=\delta_{k, j}$ and solving the resulting linear system. The limits are then given by $\zeta_{k}=\nu_{k}(0)$ and in terms of $u_{1}, u_{0}$ for either of the cases (distinct or double root) the limits turn out to be

$$
\zeta_{0}=\frac{u_{0}}{u_{1}+u_{0}+1}, \zeta_{1}=\frac{u_{1}}{u_{1}+u_{0}+1}, \zeta_{2}=\frac{1}{u_{1}+u_{0}+1} .
$$

It can be shown by CAD-computations that under the given assumptions on $u_{1}, u_{0}$ these limits are positive. Since $\zeta_{k}$ can be computed a priori from the given recurrence, $\epsilon$ above is chosen such that $\min _{k}\left(\zeta_{k}-\epsilon\right)>0$. The algorithm terminates no later than at iteration $n_{0}+m_{0}$.

REMARK 1. All CAD computations carried out in this paper were performed with Mathematica's built-in implementation of CAD [24, 25]. The computation time is negligible for all of them and could certainly be carried out with other implementations such as, e.g., [11, 4, 8] as well.

The strategies of Algorithms 1 and 3 can be combined to prove that $f(n+m) \geq 0$, for some $m \geq D$, with an extended induction hypothesis of length $D$. The proof of termination for these variations is essentially the same as the proof of Theorem 2, merely the choice of $\epsilon$ (influencing $n_{0}$ and $m_{0}$ ) has to be adapted such that all the $R_{k}\left(m_{0}, n\right)$ appearing are in an appropriate neighbourhood of the limits $\zeta_{k}$. The terminating region $I_{4}$ for Algorithm 1 with an induction hypothesis of length four is given by [21]

$$
\begin{aligned}
u_{1} & <1 \wedge u_{0}>0 \wedge 1-u_{1}+u_{1}^{2}-u_{0}>0 \\
& \wedge\left(u_{1}>0 \vee u_{1}^{2}-u_{0}-u_{1} u_{0}+u_{0}^{2}<0\right),
\end{aligned}
$$

The variant of Algorithm 3 with an extended induction hypothesis of length four extends the terminating region of
Theorem 2 by the semialgebraic set defined via

$$
-1<u_{1}<0 \wedge u_{1}^{2}<u_{0}<-\frac{1+u_{1}+u_{1}^{2}}{u_{1}} .
$$

The computational effort for doing quantifier elimination for analyzing variants extending the induction hypothesis beyond length five becomes prohibitive. However, also termination of the variation with induction hypothesis of length four implies termination of Algorithm 1 and hence as a consequence we have extended the terminating region of Algorithm 1.

Corollary 1. Algorithm 1 terminates, if either $-1<$ $u_{1}<0$ and $u_{1}^{2}<u_{0}<-\frac{1+u_{1}+u_{1}^{2}}{u_{1}}$, or $0<u_{1}<2$ and $\max \left(0, u_{1}-1\right)<u_{0}<1$.

The eigenvalues corresponding to this extension are all complex and of the form $\alpha_{1}=z_{1}+\mathbf{i} z_{2}=\overline{\alpha_{2}}$ inside the unit disk with either negative real part or

$$
0<z_{1}<\frac{1}{2} \wedge \sqrt{3} z_{1}<\left|z_{2}\right|<\sqrt{1-z_{1}^{2}} .
$$

The results obtained in this section are summarized in the figure below. The dark gray area shows the terminating region of Algorithm 3 extending the induction hypothesis up to length four. For better comparison, the previously known terminating region of Algorithm 1 with induction hypothesis up to length four is indicating by dashed lines. The remaining light gray area are the non overlapping parts of $I_{5}$ and the terminating region of Algorithm 2.

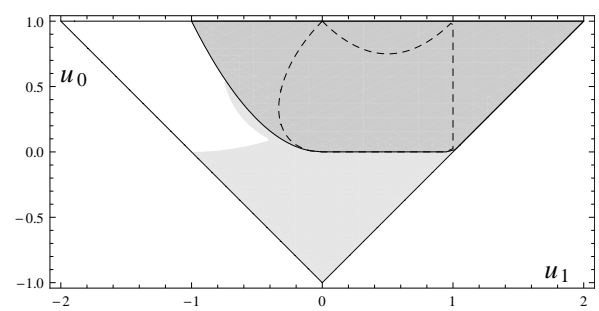

\section{ORDER FOUR AND HIGHER}

The proof of the termination result of Theorem 2 carries over immediately to higher orders. The induction step formula for a given P-finite sequence of order $d$ with a hypothesis of length $d$,

$$
\begin{aligned}
& \forall y_{0}, y_{1}, \ldots, y_{d-1} \in \mathbb{R} \forall x \in \mathbb{R}: \\
& \quad\left(x \geq 0 \wedge y_{0} \geq 0 \wedge \cdots \wedge y_{d-1} \geq 0\right) \\
& \quad \Longrightarrow r_{d-1}(x) y_{d-1}+\cdots+r_{0}(x) y_{0} \geq 0,
\end{aligned}
$$

is true, if the coefficients in the recurrence are eventually positive. Hence, following the previous discussion, to obtain a terminating region it suffices to determine the cases when the recurrence coefficients of the shifted sequence become eventually positive.

Let $\alpha_{1}, \ldots, \alpha_{\ell}, 1 \leq \ell \leq d-1$, be the distinct eigenvalues of the given recurrence and

$\chi(x)=(x-1)\left(x^{d-1}+u_{d-2} x^{d-2}+\cdots+u_{0}\right)=(x-1) U(x)$.

its characteristic polynomial. Let $\gamma_{k}(m)$ be the associated C-finite sequences defined by the recurrence (3). A closed form solution can be computed using the inverse of the generalized Vandermonde matrix. For the further reasoning 
only the limits $\zeta_{k}=\lim _{n \rightarrow \infty} \gamma_{k}(n)$ are needed and so just the coefficients of the simple eigenvalue 1 for $k=0, \ldots, d-1$ have to be computed. These values turn out to be

$$
\zeta_{k}=\frac{u_{k}}{U(1)}, \quad k=0, \ldots, d-1 .
$$

The coefficients of $U(x)$ are given by the elementary symmetric polynomials $e_{j}\left(\alpha_{1}, \ldots, \alpha_{d}\right)$ (where the eigenvalues are written with multiplicity) as

$$
u_{d-1-j}=(-1)^{j} e_{j}\left(\alpha_{1}, \ldots, \alpha_{d}\right) .
$$

If all the eigenvalues have negative real part, then all the limits (and thus eventually all the recurrence coefficients) are trivially positive. Note that in our setting complex roots may only appear in pairs of complex conjugates, since for proving positivity the sequence can only assume real values.

Corollary 2. Algorithm 3 (and thus Algorithm 1) terminates, if all eigenvalues $\alpha_{k}$ inside the interior of the unit disk have negative real part.

Combining the strategies of Algorithms 1 and 3 is in theory also possible for higher orders. In practice the analysis cannot be carried out because of computational limitations. Under the assumption that the terminating region for order $d$ is contained in the terminating region of order $d+1$ at least a guess for sufficient conditions can be computed. For recurrences of order four with an induction hypothesis of length five, such a guess could be found in reasonable time and verified easily using CAD. For this guess and provestrategy the representation

$$
\chi(x)=(x-1)\left(x^{3}+u_{2} x^{2}+u_{1} x+u_{0}\right)
$$

for the characteristic polynomial was used, because the involved inequalities as well as the output are simpler and lower in degree than using the eigenvalues. The following statement concerns only the extension beyond the cases covered in the corollary.

Theorem 3. If $0<u_{2}<1$ and $\min \left(-\frac{1}{3} u_{2}, U\right)<u_{1}<$ $\max (0, U)$ and $V_{1}<u_{0}<\min \left(u_{2}\left(1+u_{1}-u_{2}\right), V_{2}\right)$, where

$$
\begin{aligned}
U & =\frac{u_{2}^{2}\left(u_{2}-1\right)}{u_{2}^{2}-u_{2}+1}, \\
V_{1,2} & =\frac{u_{1}+u_{2}}{2} \mp \frac{\sqrt{\left(3 u_{1}+u_{2}\right)\left(u_{2}-u_{1}\right)}}{2},
\end{aligned}
$$

then Algorithm 1 terminates.

Proof. By the limit arguments used earlier it is sufficient to prove that the induction step formula for the limiting case of the recurrence coefficients holds. Let $\Omega \subset \mathbb{R}^{3}$ denote the set described by the conditions in the theorem. The eigenvalues of a recurrence with coefficients taken from $\Omega$ are a pair of complex conjugated roots and a simple real root. For recurrences of order four the limits in terms of $u_{0}, u_{1}, u_{2}$ are given by

$$
\zeta_{k}=\frac{u_{k}}{1+u_{2}+u_{1}+u_{0}}, \quad k=0,1,2,
$$

and $\zeta_{3}=1-\zeta_{2}-\zeta_{1}-\zeta_{0}$. A CAD-computation confirms

$$
\begin{aligned}
& \text { that } \\
& \begin{aligned}
\forall\left(u_{2}, u_{1}, u_{0}\right) \in \Omega \forall y_{0}, y_{1}, y_{2}, y_{3} \in \mathbb{R}: \\
y_{0}>0 \wedge y_{1}>0 \wedge y_{2}>0 \wedge y_{3}>0 \\
\quad \wedge\left(1-u_{2}\right) y_{3}+\left(u_{2}-u_{1}\right) y_{2}+\left(u_{1}-u_{0}\right) y_{1}+u_{0} y_{0}>0 \\
\quad \Longrightarrow \zeta_{3} y_{3}+\zeta_{2} y_{2}+\zeta_{1} y_{1}+\zeta_{0} y_{0}>0 .
\end{aligned}
\end{aligned}
$$

Hence, as soon as the recurrence coefficients of the sequence and of the shifted sequence are in a neighbourhood close enough to $\left(1-u_{2}\right), \ldots, u_{0}$ and $\zeta_{3}, \ldots, \zeta_{0}$, respectively, the algorithm terminates.

The terminating region was determined assuming that the complex conjugate roots are in the terminating region (5) and applying quantifier elimination using CAD to the induction step formula above with the first quantifier dropped. A further iteration of this idea extending to recurrences of order five is very space and time consuming and different strategies are needed.

Besides being hard to analyze, approaches based on CAD also (usually) do not provide insight in why the given sequence is non-negative and there is no human readable output that can be verified independently. Future directions may build on recent results on sums-of-squares representations [16] either to verify the final induction step formula or in combination with recent methods to determine a representation of a given P-finite sequence as linear combination of squares [6].

\section{REFERENCES}

[1] Horst Alzer, Stefan Gerhold, Manuel Kauers, and Alexandru Lupaş. On Turán's inequality for Legendre polynomials. Expositiones Mathematicae, 25(2):181-186, 2007.

[2] Saugata Basu, Richard Pollack, and Marie-Françoise Roy. Algorithms in Real Algebraic Geometry, volume 10 of Algorithms and Computation in Mathematics. Springer, 2nd edition, 2006.

[3] Jason P. Bell and Stefan Gerhold. On the positivity set of a linear recurrence sequence. Israel J. Math., 157:333-345, 2007.

[4] Chris W. Brown. QEPCAD B - a program for computing with semi-algebraic sets. Sigsam Bulletin, 37(4):97-108, 2003.

[5] Bob F. Caviness and Jeremy R. Johnson, editors. Quantifier Elimination and Cylindrical Algebraic Decomposition, Texts and Monographs in Symbolic Computation. Springer, 1998.

[6] Yongjae Cha. Closed form solutions of linear difference equations in terms of symmetric products. Submitted.

[7] Yongjae Cha, Mark van Hoeij, and Giles Levy. Solving recurrence relations using local invariants. In Stephen Watt, editor, Proceedings of the 2010 International Symposium on Symbolic and Algebraic Computation, ISSAC '10, pages 303-309, New York, NY, USA, 2010. ACM.

[8] Changbo Chen, Marc Moreno Maza, Bican Xia, and Lu Yang. Computing cylindrical algebraic decomposition via triangular decomposition. In Proceedings of ISSAC'09, pages 95-102, New York, NY, USA, 2009. ACM.

[9] George E. Collins. Quantifier elimination for the elementary theory of real closed fields by cylindrical 
algebraic decomposition. Lecture Notes in Computer Science, 33:134-183, 1975.

[10] George E. Collins and Hoon Hong. Partial cylindrical algebraic decomposition for quantifier elimination. Journal of Symbolic Computation, 12(3):299-328, 1991.

[11] Andreas Dolzmann and Thomas Sturm. Guarded expressions in practice. In Proceedings of ISSAC'97, 1997.

[12] Graham Everest, Alf van der Poorten, Igor Shparlinski, and Thomas Ward. Recurrence Sequences, volume 104 of Mathematical Surveys and Monographs. American Mathematical Society, 2003.

[13] Stefan Gerhold. Combinatorial Sequences: Non-Holonomicity and Inequalities. PhD thesis, RISC-Linz, Johannes Kepler Universität Linz, 2005.

[14] Stefan Gerhold and Manuel Kauers. A procedure for proving special function inequalities involving a discrete parameter. In Manuel Kauers, editor, Proceedings of ISSAC'05, pages 156-162, 2005.

[15] Stefan Gerhold and Manuel Kauers. A computer proof of Turán's inequality. Journal of Inequalities in Pure and Applied Mathematics, 7(2):\#42, 2006.

[16] Erich L. Kaltofen, Bin Li, Zhengfeng Yang, and Lihong Zhi. Exact certification in global polynomial optimization via sums-of-squares of rational functions with rational coefficients. Journal of Symbolic Computation, 47(1):1-15, 2012.

[17] Manuel Kauers. SumCracker - A Package for Manipulating Symbolic Sums and Related Objects. Journal of Symbolic Computation, 41(9):1039-1057, 2006.

[18] Manuel Kauers. Computer algebra and power series with positive coefficients. In Proceedings of FPSAC'07, 2007.

[19] Manuel Kauers. Computer algebra and special function inequalities. In Tewodros Amdeberhan and Victor H. Moll, editors, Tapas in Experimental Mathematics, volume 457 of Contemporary Mathematics, pages 215-235. AMS, 2008.

[20] Manuel Kauers and Peter Paule. A computer proof of Moll's log-concavity conjecture. Proceedings of the AMS, 135(12):3847-3856, 2007.

[21] Manuel Kauers and Veronika Pillwein. When can we detect that a P-finite sequence is positive? In Stephen Watt, editor, Proceedings of ISSAC'10, pages 195-202, 2010.

[22] Marc Mezzarobba and Bruno Salvy. Effective bounds for P-recursive sequences. J. Symbolic Comput., 45(10):1075-1096, 2010.

[23] Veronika Pillwein. Positivity of certain sums over Jacobi kernel polynomials. Advances in Applied Mathematics, 41(3):365-377, 2008.

[24] Adam Strzeboński. Solving systems of strict polynomial inequalities. Journal of Symbolic Computation, 29:471-480, 2000.

[25] Adam Strzeboński. Cylindrical algebraic decomposition using validated numerics. Journal of Symbolic Computation, 41(9):1021-1038, 2006. 